\title{
The Dynamics of Low-Perihelion Meteoroid Streams
}

\author{
Paul A. Wiegert
}

Received: 26 June 2007/ Accepted: 24 October 2007/Published online: 16 November 2007

(C) Springer Science+Business Media B.V. 2007

\begin{abstract}
The Canadian Meteor Orbit Radar (CMOR) has collected information on a number of weak meteor showers that have not been well characterized in the literature. A subsample of these showers (1) do not show a strong orbital resemblance to any known comets or asteroids, (2) have highly inclined orbits, (3) are at low perihelion distances $(\ll 1 \mathrm{AU})$ and $(4)$ are at small semimajor axes $(<2 \mathrm{AU})$. Though one might conclude that the absence of a parent object could be the result of its disruption, it is unclear how this relatively inaccessible (dynamically speaking) region of phase space might have been populated by parents in the first place. It will be shown that the Kozai secular resonance and/or PoyntingRobertson drag can modify meteor stream orbits rapidly (on time scales comparable to a precession cycle) and may be responsible for placing some of these streams into their current locations. These same effects are also argued to act on these streams so as to contribute to the high-ecliptic latitude north and south toroidal sporadic meteor sources. There remain some differences between the simple model results presented here and observations, but there may be no need to invoke a substantial population of high-inclination parents for the observed high-inclination meteoroid streams with small perihelion distances.
\end{abstract}

Keywords Meteoroid stream · Poynting-Robertson drag - Secular resonance . Toroidal meteor sources $\cdot$ Meteor shower $\cdot$ Sporadic meteors

We report here on a number of meteor showers that have been recently studied by means of the Canadian Meteor Orbit Radar (CMOR, Jones et al. 2005). These showers are weak to moderate in strength and were either discovered in the CMOR catalogue (Brown et al. 2007) or have only been poorly characterized in previous studies. In Sect. 1, those showers with clear links to parent bodies are discussed. Section 2 deals with links to other better-known showers, and Sect. 3 examines the dynamics of this ensemble of streams and its possible link to the toroidal sporadic meteor sources.

P. A. Wiegert $(\square)$

Department of Physics and Astronomy, The University of Western Ontario, London, ON,

Canada N6A 3K7

e-mail: pwiegert@uwo.ca 


\section{Links with Parent Objects}

One new shower has a clear connection to a parent. The Daytime $\varepsilon$ Perseids shower has an orbit which bears a similarity to that of comet 96P/Machholz. Table 1 lists their respective orbital elements. The Drummond (1981) $D^{\prime}$ of this association is 0.14 and the Valsecchi et al. (1999) $D$ is 0.047 though the $D$ of Southworth and Hawkins (1963) is somewhat larger at 0.435 . There is a strong resemblance in the perihelion distance $q$, inclination $i$ and longitude of the ascending node $\Omega$. The match is poorer in the semimajor axis $a$ (which is difficult to measure) and the argument of perihelion $\omega$, possibly due to precession. We conclude that this shower is likely part of the Quadrantid meteor complex, to which 96P has been linked in the past (McIntosh 1990; Babadzhanov and Obrubov 1992; Gonczi et al. 1992; Jones and Jones 1993; Jenniskens 2004; Wiegert and Brown 2005).

\section{Links with Known Streams}

Some of the other weak showers detected by CMOR are related to the multiple intersections between a meteoroid stream and the Earth's orbit that occur during the stream's precession cycle. For example, the Daytime April Piscids and the South Daytime May Arietids (sometimes called the $o$ Piscids in the literature) are both clearly related to the North and South $i$ Aquariids (see Table 2). Under apsidal precession, the intersection points of this stream with the Earth's orbit can easily be computed to occur near values of the argument of perihelion $\omega$ of $50^{\circ}, 130^{\circ}, 230^{\circ}$ and $310^{\circ}$. We have also verified this by numerical experiment. Thus the Daytime April Piscids and the South Daytime May Arietids, together with the N/S $i$ Aquariids, complete the set of four separate showers produced by the precession of meteoroids released from a single parent.

\section{The Remaining Streams}

Despite the associations discussed in the two preceding sections, most of the weak showers in the CMOR catalog do not have immediately obvious parent bodies, nor clear links to known streams. In fact, many of these streams have semimajor axes $a$ below 2 AU, perihelia $q$ well inside Mercury's orbit, and high inclinations (Table 3 and Fig. 1), placing them in a region of phase space that is very sparsely populated by comets and asteroids. A search of the asteroid and comet databases turns up no bodies with orbits clearly similar to those of these streams.

One might speculate that the low-perihelion distances of these streams, together with the high activity levels and rapid depletion they would produce in a source comet, might account for the current absence of parent bodies. The parents would simply have disrupted or become inactive or extinct. However this would not explain how the source bodies

Table 1 Comparison of the orbits of 96P/Machholz (Marsden and Williams 2005) and the Daytime $\varepsilon$ Perseids

\begin{tabular}{lllllll}
\hline Name & $a(\mathrm{AU})$ & $q(\mathrm{AU})$ & $e$ & $i\left(^{\circ}\right)$ & $\Omega\left({ }^{\circ}\right)$ & $\omega\left(^{\circ}\right)$ \\
\hline $\mathrm{D} \varepsilon$ Perseids & $4.6 \pm 1$ & $0.13 \pm 0.01$ & $0.97 \pm 0.01$ & $63 \pm 2$ & $96 \pm 0.3$ & $40 \pm 2$ \\
96P/Machholz & 3.01 & 0.123 & 0.959 & 59.9 & 94.5 & 14.6 \\
\hline
\end{tabular}

Errors for the shower elements are approximate 
Table 2 The elements of the Daytime April Piscids and South Daytime May Arietids, together with those of the better-known North and South $i$ Aquariids

\begin{tabular}{llllllr}
\hline Name & $a(\mathrm{AU})$ & $q(\mathrm{AU})$ & $e$ & $i\left({ }^{\circ}\right)$ & $\Omega\left({ }^{\circ}\right)$ & $\omega\left(^{\circ}\right)$ \\
\hline Daytime April Piscids & 1.51 & 0.26 & 0.83 & 4.7 & 25 & 50 \\
S Daytime May Arietids & 1.51 & 0.27 & 0.82 & 5.1 & 227 & 232 \\
$\mathrm{~N} i$ Aquariids & 1.52 & 0.27 & 0.83 & 5.7 & 159 & 309 \\
$\mathrm{~S} i$ Aquariids & 1.55 & 0.22 & 0.86 & 5.3 & 309 & 134 \\
\hline
\end{tabular}

The orbits are from the CMOR catalogue

Table 3 A selection of the new or previously little-studied meteor showers in the CMOR catalogue

\begin{tabular}{|c|c|c|c|c|c|c|}
\hline Name & $a(\mathrm{AU})$ & $q(\mathrm{AU})$ & $e$ & $i\left(^{\circ}\right)$ & $\Omega\left({ }^{\circ}\right)$ & $\omega\left(^{\circ}\right)$ \\
\hline N Daytime $\omega$ Cetids & 1.58 & 0.12 & 0.93 & 34 & 45 & 33 \\
\hline S Daytime $\omega$ Cetids & 1.72 & 0.14 & 0.92 & 36 & 225 & 216 \\
\hline S June Aquilids & 1.12 & 0.06 & 0.94 & 56 & 260 & 159 \\
\hline Daytime $\gamma$ Taurids & 1.57 & 0.10 & 0.93 & 23 & 266 & 211 \\
\hline Vulpeculids & 0.76 & 0.17 & 0.77 & 55 & 105 & 335 \\
\hline N June Aquilids & 1.71 & 0.11 & 0.94 & 39 & 101 & 328 \\
\hline$\beta$ Equulids & 0.89 & 0.16 & 0.82 & 50 & 106 & 330 \\
\hline July $\sigma$ Cassiopeiids & 1.09 & 1.00 & 0.08 & 81 & 105 & 217 \\
\hline$\psi$ Cassiopeiids & 2.14 & 0.93 & 0.56 & 83 & 118 & 141 \\
\hline $\mathrm{N} \delta$ Aquariids & 1.81 & 0.10 & 0.95 & 24 & 139 & 329 \\
\hline$\sigma$ Serpentids & 1.92 & 0.16 & 0.92 & 64 & 276 & 41 \\
\hline$\omega$ Serpentids & 1.37 & 0.16 & 0.88 & 56 & 276 & 39 \\
\hline$\theta$ Coronae Borealids & 1.11 & 0.92 & 0.17 & 77 & 296 & 125 \\
\hline$\lambda$ Bootids & 1.49 & 0.96 & 0.36 & 79 & 295 & 207 \\
\hline$\zeta$ Coronae Borealids & 2.34 & 0.82 & 0.65 & 80 & 294 & 125 \\
\hline$\alpha$ Antilids & 2.47 & 0.14 & 0.94 & 64 & 136 & 140 \\
\hline
\end{tabular}

reached these orbits in the first place, as the dynamical evolution of bodies into this region is slow.

We report here that Poynting-Robertson (PR) drag is likely responsible for the current orbits of these showers. It will be shown that streams produced by comets at larger $a$ and $q$ can evolve into streams of the type described above (or at least the smaller members of these streams can) on time scales of only thousands of years, short compared to their precession times.

Additionally, we report that many such streams are trapped in the Kozai resonance (Kozai 1962) which causes their eccentricities $e$ and inclinations $i$ to oscillate. Such meteoroids produce radiant distributions with some of the characteristics of the toroidal sporadic meteor sources.

\subsection{Investigations}

In order to study the dynamics of these streams, the showers in Table 3 were simulated numerically with a symplectic Wisdom and Holman (1991) style integrator able to handle 
Fig. 1 The orbital distributions of near-Earth asteroids (dots, from the AstDys website http://hamilton.dm.unipi.it/ cgi-bin/astdys/astibo), comets (black circles, Marsden and Williams (2005)) and the showers discussed here (grey diamonds) in (a) $a-e$ and (b) $e-i$ space
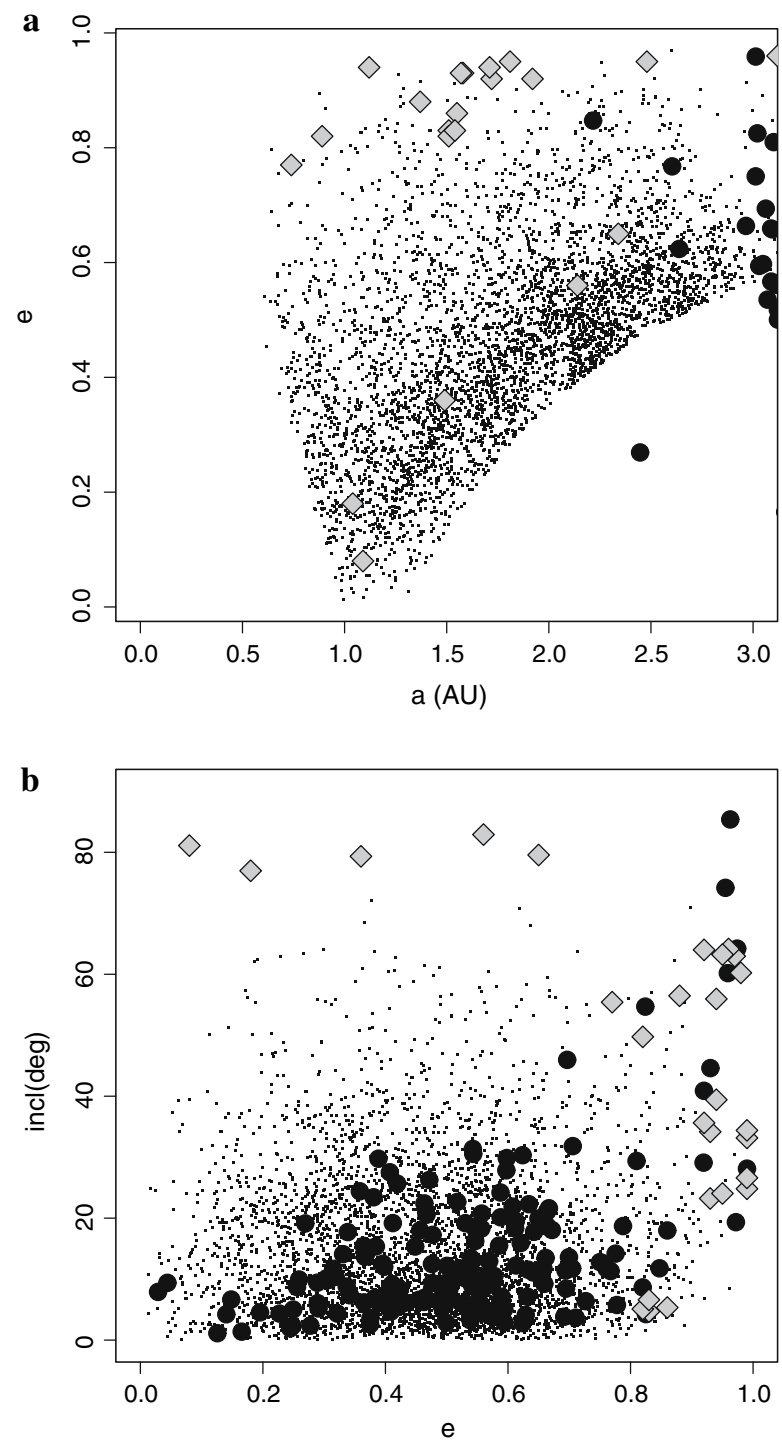

close encounters by the hybrid method (Chambers 1999). Two sets of ten particles were spread along the orbit of each meteoroid stream at equal intervals of mean anomaly. One set was assigned a beta of zero for comparison purposes. The other set was assigned a $\beta$ value of 0.0057 to simulate particles of a density of $2,000 \mathrm{~kg} \mathrm{~m}^{-3}$ and a radius of $100 \mu \mathrm{m}$ (Weidenschilling and Jackson 1993). Each set was integrated backwards for 50,000 years with a time step of one day.

The simulation of multiple particles per stream allows us to better understand the effects of differential perturbations such as planetary encounters. However, these simulations have only a small number of particles and are not of the caliber of those frequently used these days for detailed shower timing and strength predictions, which may involve tens of 
thousands or more particles. Nevertheless they provide great insight into the dynamical behaviour of these streams.

A common feature of the numerical simulations is a substantial change in the semimajor axes of the stream orbits over time. Some streams can undergo changes in $a$ at rates exceeding $1 \mathrm{AU}$ per $10^{3}$ years, though average rates near $1 \mathrm{AU}$ per $10^{4}$ years are more typical. Thus the stream produced by a Jupiter family comet with $a \approx 3$ AU could become one with $a \sim 1$ AU (like many of the showers in Table 3 ) in only a few 1,000 years.

An example of the semimajor axis evolution of one such stream, the $\beta$ Equulids, is shown in Fig. 2. Note how the particles with $\beta=0.0057$ have rapidly changing semimajor axes while the control particles with $\beta=0$ remain largely unaffected. This indicates that these changes are indeed the result of radiation forces. If the new showers discussed here are primarily composed of small particles, then they could have been released from comets with larger values of $a$ and $q$ and subsequently transported to their current orbits by PR drag. This might also explain the absence of these showers from visual shower catalogues, as such streams are unlikely to contain many of the larger meteors (with smaller $\beta$ values) which are more easily observed by optical means.

Figure 3 shows the eccentricity evolution of the $\beta$ Equulids stream. Notably absent is the monotonic circularization expected for meteoroids experiencing strong PR drag (Wyatt and Whipple 1950), though we note that a careful treatment by Breiter and Jackson (1998) revealed that there were cases where a small increase in $e$ could be expected from PR drag. In the simulations presented here, $e$ is seen to oscillate on time scales of $10^{4}$ years. The reason that an alternation of $e$ occurs rather than a simple reduction in its value is because of the action of the Kozai resonance (Kozai 1962), also known as the secular precession effect discussed by Babadzhanov and Obrubov (1987). This secular effect pumps angular momentum in and out of the meteoroid orbit faster than PR drag removes it, and thus controls the value of $e$ in this dynamical regime.

The secular resonance that affects $e$ also produces an oscillation in the inclination $i$. Its effect on the $\beta$ Equulids stream is shown in Fig. 4. Inclination and eccentricity oscillate out of phase with each other, and the meteoroids spend much of their time at high inclination,

Fig. 2 The evolution of the semimajor axis of the $\beta$ Equulids meteoroids simulated backwards for 50,000 years. The open circles are $100 \mu \mathrm{m}$ radius particles $(\beta=0.0057)$, while the crosses are particles with $\beta=0$

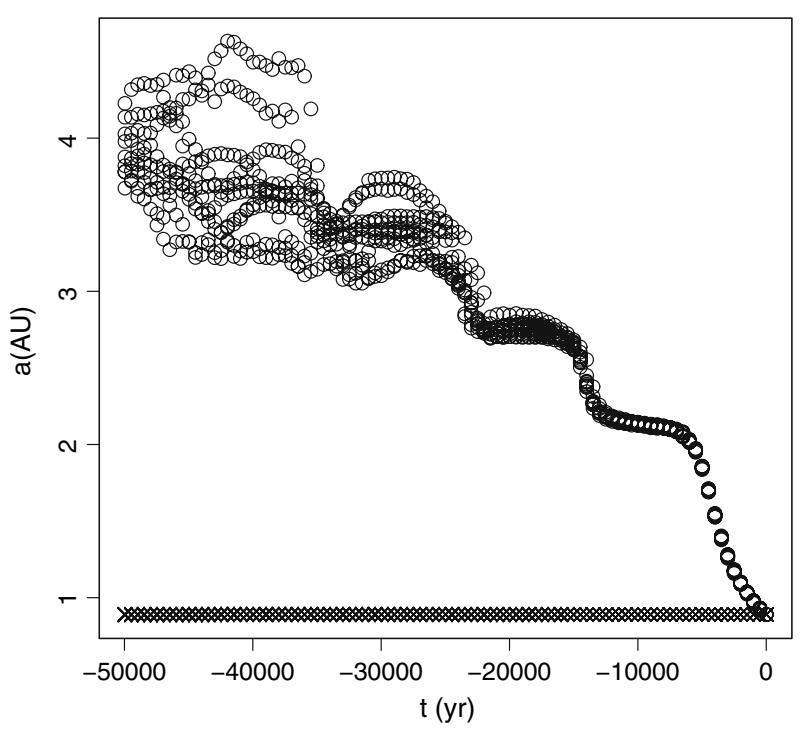


Fig. 3 The evolution of eccentricity of the $\beta$ Equulids meteoroids simulated backwards for 50,000 years. See Fig. 2 for more details

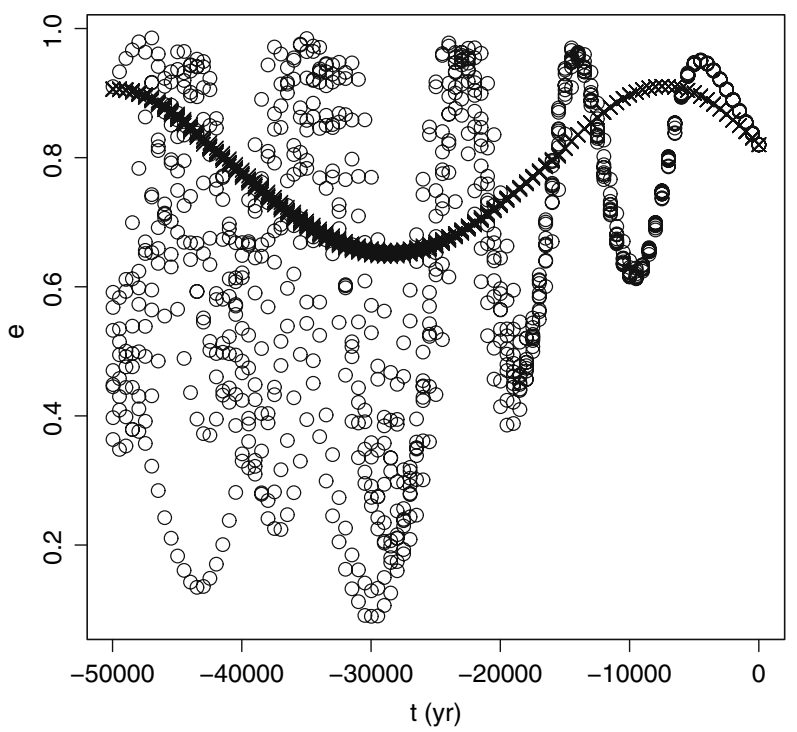

at a time-average value near $60^{\circ}$. Thus meteoroid streams produced at much lower inclination $\left(\lesssim 20^{\circ}\right)$ can be driven up to much higher inclination $\left(>r \operatorname{sim} 80^{\circ}\right)$ by this effect. In fact, these particles spend most of their time at high inclination. This result is relatively insensitive to $\beta$, even particles at $\beta=0$ also have large time-averaged inclinations. Thus, there is no need to invoke a substantial population of high-inclination parents for these streams; they could easily be produced by bodies with a much flatter distribution (e.g. the Jupiter-family comets) pumped up by the secular resonance.

The high time-averaged inclination of these meteoroids also suggests a connection with the north and south toroidal sporadic sources that we investigate next.

Fig. 4 The evolution of inclination of $\beta$ Equulids meteoroids simulated backwards for 50,000 years. See Fig 2 for more details

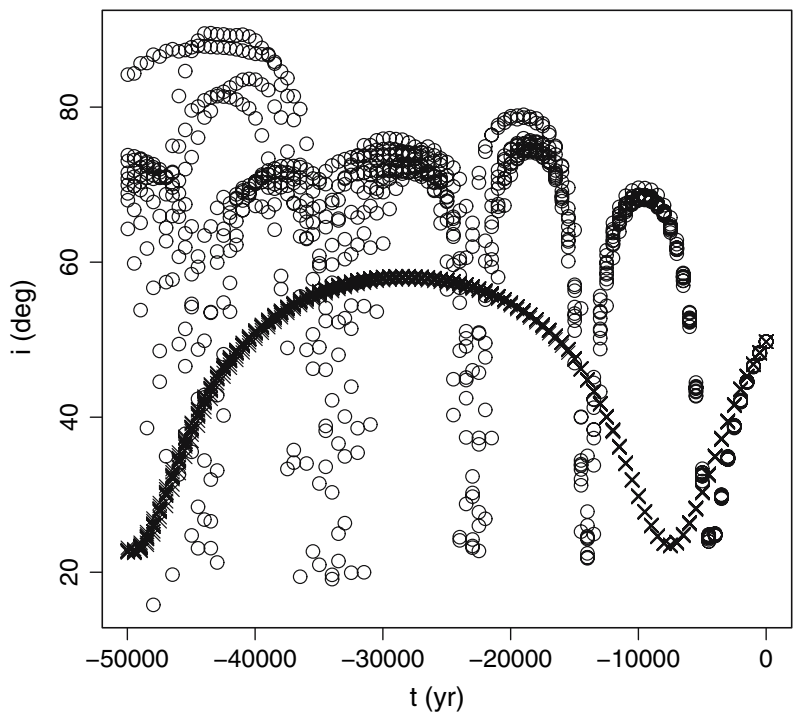




\subsection{The Toroidal Sporadic Meteor Sources}

The orbital element distributions of the north toroidal sporadic source have been determined (e.g. Jones and Brown 1993), and it is expected that those of the southern toroidal source will be similar. However, the origin of the meteors that produce these sources is not known. The elements presented in Jones and Brown (1993) for the northern source show a peak in $a$ at $1 \mathrm{AU}$, one in inclination near $60^{\circ}$, and a distribution in $e$ with a preponderance of near-circular orbits. The high-inclination is particularly puzzling owing to the absence of comets or asteroids on such orbits. Could the high inclination showers discussed here be connected to the toroidal sporadic sources? Perhaps as these meteoroids diffuse away from the shower orbits and drift inwards under PR drag, many remain in the secular resonance at high $i$, ultimately becoming toroidal sporadics?

In order to investigate this possibility, we simulated meteoroid streams originating from hypothetical parents of the high- $i$ streams described above. The difference between these simulations and the ones mentioned earlier are (1) these simulations are run forwards in time, (2) three different particle radii are included: 50, 100 and $200 \mu \mathrm{m}$ (10 particles each, with appropriate $\beta$ values) and (3) the meteoroid streams are started with the elements given in Table 3 with the exception that the semimajor axis is set to 3 AU. This provides a proxy for the putative cometary parents of these streams, here assumed to be Jupiter-family comets. By simulating these streams forwards under PR drag, we can make a rough determination of whether or not the meteoroids produced by such parents could produce the toroidal sporadic sources.

Figure 5 shows the resulting density of radiants of the simulated meteoroids with nodes within $0.1 \mathrm{AU}$ of the Earth over $10^{5}$ years (roughly their collisional lifetime (Grun et al. 1985), though their high inclinations are likely to prolong their survival in practice, weighted according to their collision probability with the Earth (from Opik (1951) as given by Galligan and Baggaley (2004)). The radiants are based on the true minimum approach distance between the orbits, not just the distance between the nodes. The radiants are

Fig. 5 The radiant distribution of simulated meteoroids weighted according to the collision probability with the Earth. Darker tones indicate a higher density of meteor radiants. The Earth's apex is towards the origin in this plot and the Sun is at a relative longitude of $-90^{\circ}$

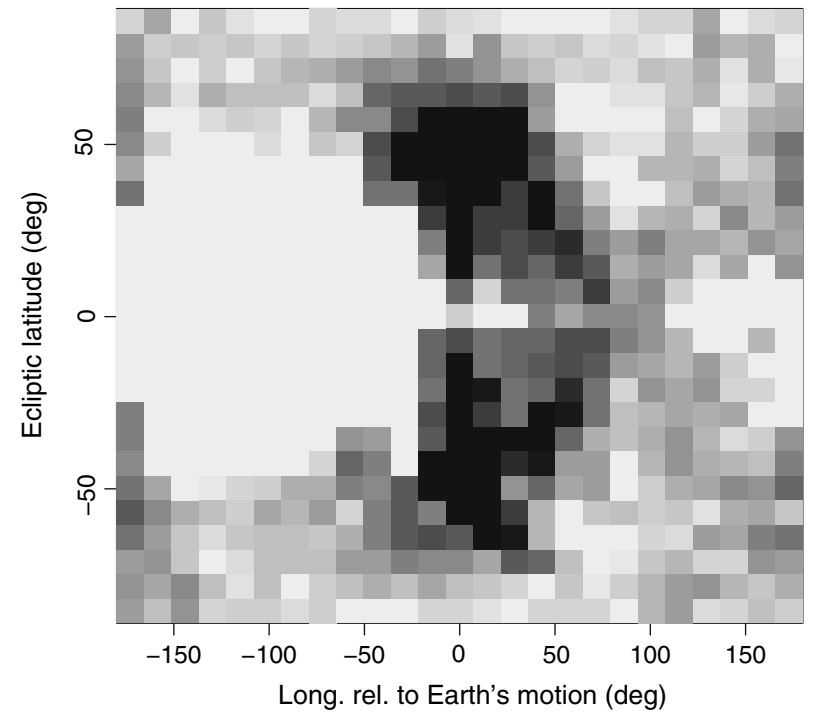


determined simply from the relative velocity of the meteoroid and the Earth at closest approach.

Both north and south toroidal radiants are reproduced, though they are nearer the ecliptic plane than the observed toroidal radiants which are at ecliptic latitudes of $\pm 60^{\circ}$ (Jones and Brown 1993). The orbital distributions of meteors within the toroidal radiant will be examined next. The orbits will be found to bear some resemblance to observed toroidal meteors, but this scenario probably does not provide a complete explanation of the origin of the toroidal sources.

Figure 6 shows the distribution of inclinations within the radiant area defined by a longitude relative to the apex of less than $30^{\circ}$ and a latitude (either north or south) between
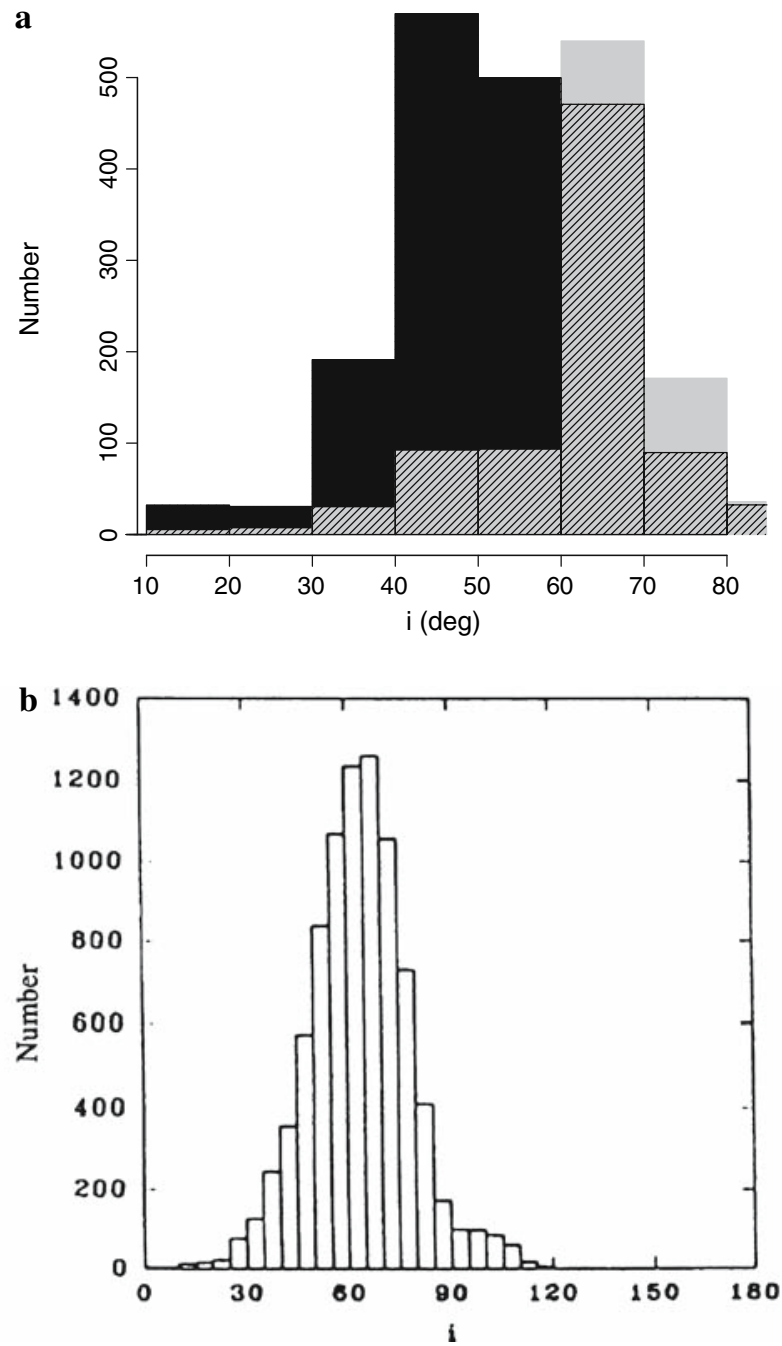

Fig. 6 (a) The distribution of the inclinations of simulated meteoroids accumulated over $10^{5}$ years. The histogram in grey is unweighted; the black is weighted according to the collision probability with the Earth, normalized to a similar peak value. Panel (b) is the observed distribution of north toroidal source meteors from Jones and Brown (1993) 
Fig. 7 The distribution of semimajor axes of (a) simulated meteoroids and (b) observed north toroidal source meteors from Jones and Brown (1993). See Fig. 6 for more details
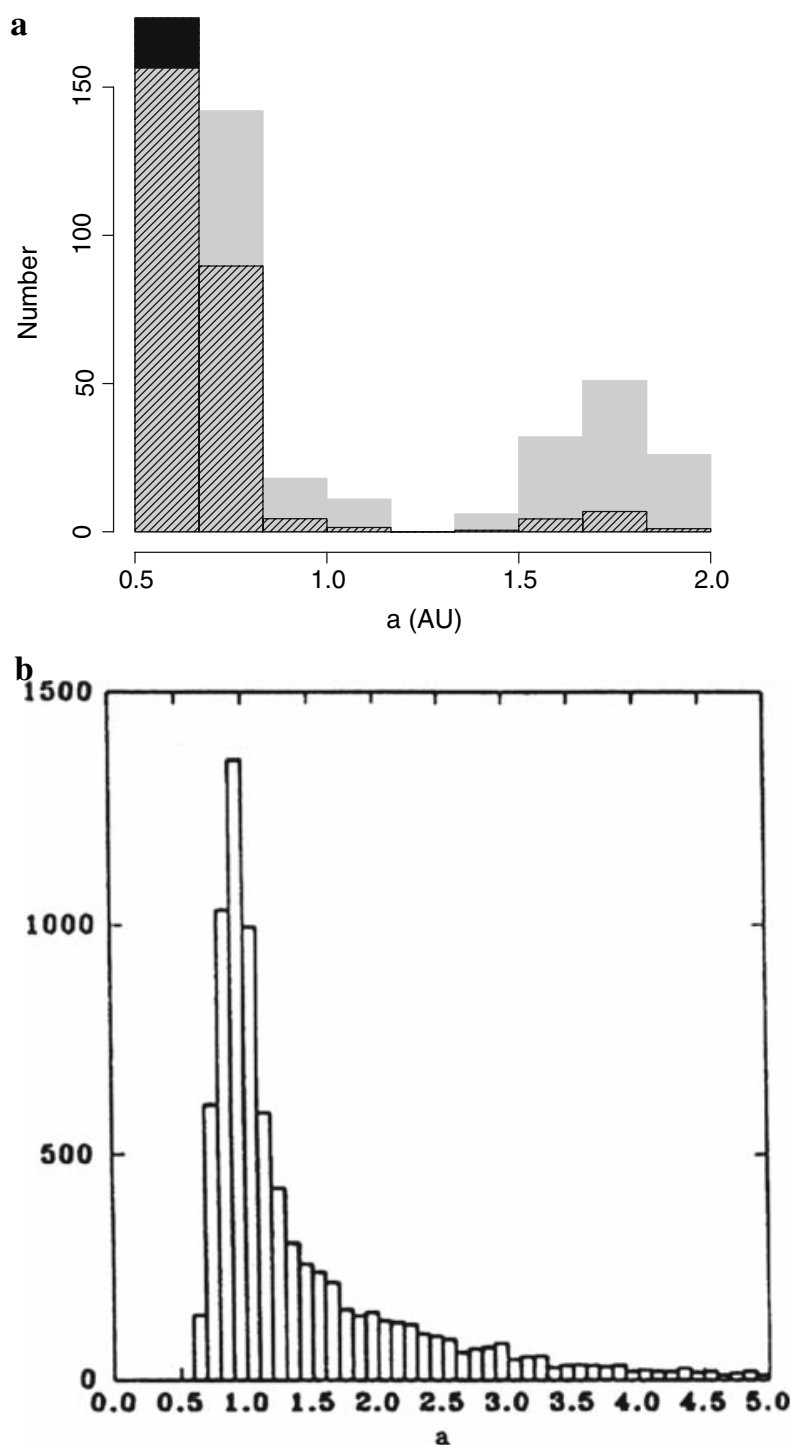

$40^{\circ}$ and $70^{\circ}$. The distribution shows a peak at high inclination, similar to that observed but not expected given our choice of radiant latitude. Figures 7 and 8 show the orbit element distributions for the semimajor axis and eccentricity for those meteoroids in the above radiants. The weighted distributions bear some resemblance the measured distributions for the north toroidal source, given in Fig. 9 of Jones and Brown (1993), but are not identical. The simulated semimajor axis distributions, both weighted and unweighted, are sharply peaked like the observations, but at values below those of the observed distribution. The simulated and observed eccentricity distributions differ as well. The observed distribution contains a preponderance of near-circular orbits. The unweighted simulated distribution is 

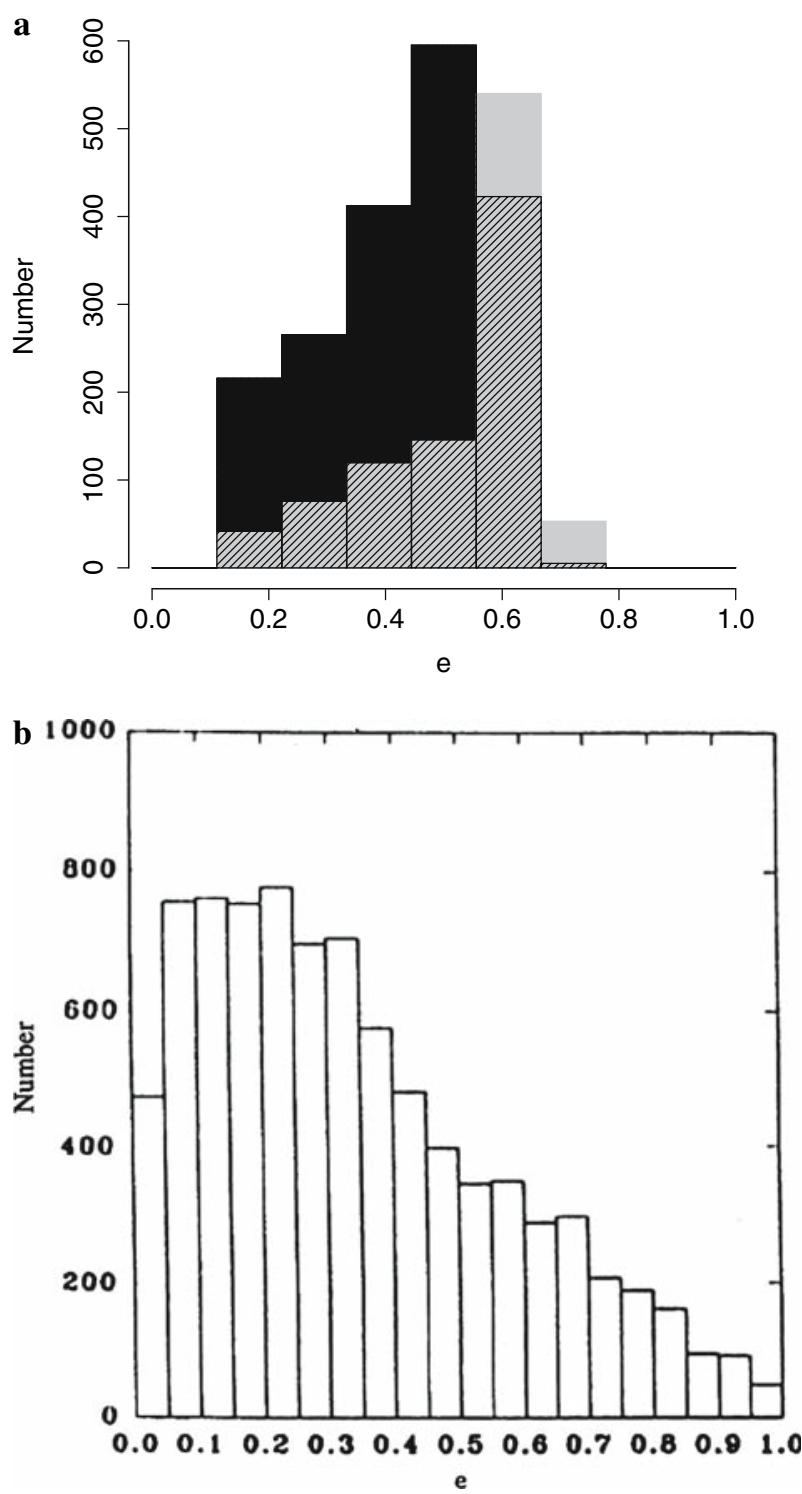

Fig. 8 The distributions of eccentricity of (a) simulated meteoroids and (b) observed north toroidal source meteors from Jones and Brown (1993). See Fig. 6 for more details

peaked near $e=0.6$. Though the discrepancy is less pronounced for the weighted distribution (which should more closely match observations), the match is far from perfect. The differences between the experimental and theoretical distributions may simply be due to our coarse modelling of the parent streams. However, it probably also indicates that the crude scenario employed here, despite some intriguing intimations, is insufficient to completely explain the toroidal sporadic sources. 


\section{Conclusions}

The CMOR catalogue of meteor shower orbits provides much new information on the nature of meteoroid streams near the Earth, particularly improved orbits for many weak showers. Using this information, the Daytime $\varepsilon$ Perseids can now be connected with comet 96P/Machholz. Two other poorly-characterized showers, the Daytime April Piscids and South Daytime May Arietids, can now be linked to the North and South $i$ Aquariids. As well, a number of unusual weak showers with small $q$ and $a$ and large values of $i$ were shown to be consistent with small $(r \sim 100 \mu \mathrm{m})$ meteoroids released from comets with much larger $a$, and evolving under PR drag and the Kozai resonance. Initial indications are that these meteoroids eventually become part of the north and south toroidal sources, though more work is needed.

Acknowledgements PW extends his thanks to J. Vaubaillon for presenting these results at the Meteoroids 2007 conference in Barcelona in the author's absence, as well as to J. M. Trigo-Rodríguez and the LOC for allowing the last-minute replacement. This work was supported by the Natural Sciences and Engineering Research Council of Canada.

\section{References}

P.B. Babadzhanov, I.V. Obrubov, Evolution of meteoroid streams, in European Regional Astronomy Meeting of the IAU, eds. by Z. Ceplecha, P. Pecina, vol. 2 (Czechoslovak Academy of Sciences, Ondrejov, Czechoslovakia, 1987), pp. 141-150

P.B. Babadzhanov, Y.V. Obrubov, P/Machholz 1986 VIII and Quadrantid meteoroid stream. Orbital evolution and relationship, in Asteroids, Comets, Meteors 1991, eds. by A. Harris, E. Bowell (University Arizona Press, Tucson, 1992), pp. 27-32

S. Breiter, A.A. Jackson, Unified analytical solutions to two-body problems with drag, MNRAS 299, 237243 (1998)

P. Brown, R.J. Weryk, D.K. Wong, J. Jones, The Canadian Meteor Orbit Radar (CMOR) Meteor Shower Catalogue, Earth Moon Planets, this issue (2007). doi:10.1007/s11038-007-9162-6

J.E. Chambers, A hybrid symplectic integrator that permits close encounters between massive bodies, MNRAS 304, 793-799 (1999)

J.D. Drummond, A test of comet and meteor shower associations. Icarus 45, 545-553 (1981)

D.P. Galligan, W.J. Baggaley, The orbital distribution of radar-detected meteoroids of the Solar system dust cloud. MNRAS 353, 422-446 (2004). doi:10.1111/j.1365-2966.2004.08078.x

R. Gonczi, H. Rickman, C. Froeschlé, The connection between Comet P/Machholz and the Quadrantid meteors, MNRAS 254, 627-634 (1992)

E. Grun, H.A. Zook, H. Fechtig, R.H. Giese, Collisional balance of the meteoritic complex, Icarus 62, 244272 (1985). doi:10.1016/0019-1035(85)90121-6

P. Jenniskens, 2003 EH1 is the Quadrantid shower parent comet, Astron. J. 127, 3018-3022 (2004)

J. Jones, P. Brown, Sporadic meteor radiant distributions-orbital survey results, MNRAS 265, 524-532 (1993)

J. Jones, W. Jones, Comet Machholz and the Quadrantid meteor stream, MNRAS 261, 605-611 (1993)

J. Jones, P. Brown, K.J. Ellis, A.R. Webster, M. Campbell-Brown, Z. Krzemenski, R.J. Weryk, The Canadian Meteor Orbit Radar: system overview and preliminary results, Plan Space Sci. 53, 413-421 (2005)

Y. Kozai, Secular perturbations of asteroids with high inclination and eccentricity, Astron. J. 67, 591-598 (1962)

B.G. Marsden, G.V. Williams, Catalogue of Cometary Orbits, 16th edn. (IAU Central Bureau for Astronomical Telegrams-Minor Planet Center, Cambridge, 2005)

B.A. McIntosh, Comet P/Machholtz and the Quadrantid meteor stream, Icarus 86, 299-304 (1990)

E.J. Opik, Collision probability with the planets and the distribution of planetary matter, Proc. R. Ir. Acad. Sect. A 54, 165-199 (1951)

R.B. Southworth, G.S. Hawkins, Statistics of meteor streams, Smithsonian Contrib. Astrophys. 7, 261-285 (1963) 
G.B. Valsecchi, T.J. Jopek, C. Froeschle, Meteoroid stream identification: a new approach-I. Theory, MNRAS 304, 743-750 (1999)

S.J. Weidenschilling, A.A. Jackson, Orbital resonances and Poynting-Robertson drag, Icarus 104, 244-254 (1993)

P. Wiegert, P. Brown, The Quadrantid meteoroid complex, Icarus 179, 139-157 (2005). doi:10.1016/j. icarus.2005.05.019

J. Wisdom, M. Holman, Symplectic maps for the $n$-body problem, Astron. J. 102, 1528-1538 (1991)

S.P. Wyatt, F.L. Whipple, The Poynting-Robertson effect on meteor orbits, Astrophys. J. 111, 134-141 (1950) 